\title{
Is Fengshui Science or Superstition? A New Criterion for Judging the Value of Knowledge Systems
}

\author{
ZHANG Yong-feng, DAI Wei \\ Tsinghua University, Beijing, China
}

\begin{abstract}
Fengshui, which can be translated as Wind-Water literally in English, is an ancient Chinese system of laws considered to govern spatial arrangement and orientation in relation to the flow of $Q i$, and whose favorable or unfavorable effects are taken into account when sitting and designing buildings. Similar systems exist in many other cultures such as Vastu Shastra in India, which consists of precepts born out of a traditional and archaic view on how the laws of nature affect human dwellings. Although prospered in ancient society, modern reactions to Fengshui are mixed. The Skeptic Encyclopedia of Pseudoscience states that principles of Fengshui are quite rational, but folk remedies and superstitions have been incorporated into its eclectic mix. In this paper, we do not distinguish Fengshui and other similar systems between science and superstition, but try to propose a criterion for judging whether a knowledge system is valuable, and if so, to whom it is valuable. We will end up arguing that, a knowledge system satisfying the criterion of relatively true property is valuable at least to its community of believers, and the problem of whether a knowledge system has greater value is essentially a problem of whether it is relatable to other knowledge systems, so as to expand its community of believers.
\end{abstract}

Keywords: philosophy of science, superstition, Fengshui, relatively true property

\section{Introduction}

Philosophy of science has long been an important research area for philosophers, which has also made great impacts on the development of science and technology. Karl Popper contented that the central question in the philosophy of science is distinguishing science from non-science (Thornton, 2006). We are here mainly focused on science and superstitions, with the latter broadly considered to be a typical kind of non-science.

Science is usually described as a systematic enterprise that builds and organizes knowledge in the form of testable explanations and predictions about the universe. As described in Merriam-Webster Online Dictionary, science is knowledge or a system of knowledge covering general truths or the operation of general laws, especially as obtained and tested through scientific methods. More precisely, a knowledge system is referred to as such a system that has one or more axioms or hypotheses (propositions made as bases for reasoning without proving their truth), as well as a set of reasoning rules, together with all the conclusions based on these

\footnotetext{
*Acknowledgements: The authors thank LIU Li, Professor at School of Humanities, Tsinghua University, and FENG Shi, M.S. at School of Information Science, Tsinghua University, for the fruitful discussions with them.

ZHANG Yong-feng, M.S. at Institute of Artificial Intelligence, Tsinghua University.

DAI Wei, M.S. at King Abdullah University of Science and Technology, Tsinghua University.
} 
hypotheses and rules. Note that a knowledge system is not necessarily a deterministic system, which requires that no randomness be involved in the development of future states of the system. A knowledge system only requires two basic elements: hypotheses and rules. Superstition, on the other hand, is defined as a widely held but unjustified belief in supernatural causation leading to certain consequences of an action or event, or a practice based on such a belief.

The process through which conclusions are arrived at has drawn much attention when distinguishing science from superstition (Shermer, 2002, pp. 111-112). According to broadly accepted perspectives, an important factor to consider when distinguishing science from superstition is the role that skepticism plays when people arrive at conclusions. Science demands people to keep a skeptical attitude anytime and anywhere in practice. One is demanded to keep analyzing and critical thinking before accepting any conclusions, let alone accepting conclusions that can not or have not been confirmed. Even for those conclusions that have been confirmed and utilized in practice, one should also try to examine their limits in space and time, and the accuracy of observations related with these conclusions. On the contrary, superstition is such a belief that has no basis or only a few unjustified observations.

As the significant difference between the processes through which conclusions are made, philosophers tend to put science and superstition in contrary places: One prefers to trust those objects, conclusions, and experiences which are confirmed through accurate scientific methods, and utilizes them in practice with confidence. One tends to doubt their value and refuses to utilize them in practice if they are not verified through scientific methods but ways such as simple or limited observations.

However, it is not always proper for us to judge the value of objects, conclusions, and experiences in such a way, as it brings about two essential problems. First, it is not guaranteed to be absolutely true even if a conclusion is drawn through scientific methods. Second, it is not guaranteed to be absolutely wrong even if a conclusion is drawn through methods that are not scientific or not scientific enough. As a result, judging the value of a conclusion only by the process through which we arrive at the conclusion is not enough, and even may not be reasonable. We need criterions that are more objective and practical to decide whether a conclusion or a knowledge system is valuable, and if so, to whom it is valuable. Further more, we need to know what on earth is the essence of the value of a knowledge system.

In this paper, we devote ourselves to proposing a criterion for judging the value of a knowledge system. Note that what we are discussing here is the value of a knowledge system, namely, whether and to whom a knowledge system is valuable. In other words, we will not discuss the problem of whether a knowledge system is science or not, and more precisely speaking, there is no need for us to discuss this problem with the criterion to be proposed. In fact, we are not going to make a distinction between science and non-science in this paper, but to show that we can still judge whether a knowledge system is valuable without the concept of science and non-science, and the so-called science or not problem is in fact a problem of whether knowledge systems are relatable ${ }^{1}$ to each other.

\footnotetext{
${ }^{1}$ A knowledge system is relatable to another knowledge system if there exists at least one method to transform conclusions in the first system to conclusions in the second system. The first system is partially relatable to the second system if part of its conclusions can be transformed to the second one. Two knowledge systems are relatable to each other if this property holds in two directions. For example, geometry and algebra are relatable to each other, because the conclusions can be transformed from geometric forms to algebraic forms as well as reversely, through Cartesian coordinate system.
} 


\section{Fengshui and Mathematics}

Fengshui is most extensively investigated in Zangshu (Book of Burial) by GUO Pu of the Jin Dynasty. In this section, we make a comparison of Fengshui together with a typical example, mathematics, in order to introduce the basic ideas of this paper.

We will start with the question why people trust mathematics. Science, in narrow sense, is defined as the intellectual and practical activity encompassing the systematic study of the structure and behavior of the physical and natural world through observations and experiments, which is also usually referred to as natural science. From this perspective of view, science is a set of laws created by God, which is not changeable. As a result, mathematics is usually not classified as science ${ }^{2}$. In fact, as a course studying quantity, space, structure, and change, mathematics is an invention or partially an invention of human beings ${ }^{3}$. Mathematics is a self-consistent knowledge system based on several axioms and reasoning rules. In mathematics, the reason why we trust a conclusion, namely, we call it a theorem, is that it can be derived through generally accepted reasoning rules on basis of generally accepted axioms. In other words, we can prove it. If a conclusion has not been proved yet, it is called a conjecture rather than a theorem.

One can see that the essential reason for our belief in mathematics is that we identify the same axioms and follow the same reasoning rules. This is the very reason for us to believe the conclusions derived by others who also identify those axioms and reasoning rules. We are sure that for any person who follows the same axioms and rules, he or she must arrive at the same conclusions, which is mathematically repeatable ${ }^{4}$. As a result, people do not doubt the exactness and soundness of mathematics, as long as they identify those axiom bases and reasoning rules, which further leads to their acceptance of all the conclusions derived according to the axioms and rules.

But people tend to take different attitudes when considering Fengshui or Vastu Shastra, and in most cases they are viewed as superstitions. In fact, such an attitude is mainly rooted in the doubt of the process through which Fengshui is proposed. In ancient China, Fengshui used to be an important set of principles to judge whether a natural environment is good for people or not. It was widely used to determine the places and orientations for palaces, villages, or tombs. But with the rapid development of experiment-based courses such as physics, chemistry, and biology in modern years, people begin to doubt those principles described in Fengshui. An important pattern for doing research in modern natural science is evaluation, interpretation, observation, conjecture, experiment, and assessment. A conjecture will not be universally accepted unless it is confirm by strict experiments. Otherwise, such conjectures will always be conjectures, waiting to be confirmed, or to be discarded. From this point of view, many of the principles and rules in Fengshui are still conjectures, which make

\footnotetext{
${ }^{2}$ Of course, this is still a philosophically open problem with various viewpoints. The authors just take the argument that mathematics should not be classified into science here. For much more detailed information on this problem, the book The Foundations of Mathematics: A Study in the Philosophy of Science is a good choice.

3 This is also a problem debated for centuries with two basic arguments: Geek Philosophy Plato's Platonic Theory, which states that mathematics is discovered, and a counterclaim by philosopher Mark Belaguer's theory. But it has been broadly accepted that mathematics is at least partially invented. For more information, Kuhn Robert Lawrence's Is Mathematics Invented or Discovered (Science and Religion Today, 1 Apr. 2010) is a good reference.

${ }^{4}$ Repeatability is an important concept in the problems discussed in this paper. Conclusions in a knowledge system are repeatable if people can arrive at the same conclusions anytime and anywhere, as long as they identify the same axioms or hypotheses, and obey the same reasoning rules of this system. Mathematical repeatability is a kind of absolute repeatability. In fact, the only thing that one needs to do is just writing the proof of a conclusion on a piece of paper. Another kind of repeatability is experimental repeatability, which requires experimental results are repeatable given the same conditions and experimental methods.
} 
people keep doubtful attitudes towards them.

In fact, Fengshui is similar to Mathematics in that they can both be viewed as human-made, or partially human-made knowledge systems. In mathematics, it is because people identify the same axioms and obey the same reasoning rules that they can arrive at the same conclusions even though they are at different times and in different places. Similarly, if we identify the same basic concepts and obey the same reasoning rules in Fengshui, we can also come into the same conclusions with our ancestors in ancient years, without caring about whether these conclusions are right or not ${ }^{5}$. By this means, we will be able to understand their thoughts and behaviors, which helps us to study them better and make a guess at their potential behaviors more correctly. Such a method is playing a very important role in archeology and history studies.

It is not reasonable to simply view a knowledge system as a kind of superstition when it does help us to understand existing phenomena and to predict potential phenomena, just like we cannot view mathematics as superstitions. We will begin from this basic observation on, and discuss the role that human-made knowledge systems play in helping us to understand the universe. Further more, we will discuss the relationship between science and superstition, as well as when and how they can be transformed into each other.

\section{A Criterion for Judging Whether a Knowledge System Is Valuable}

Nature and universe are objects, which are so complicated and recondite, that it is practically very difficult for human beings to acquire all the knowledge about them without making any mistakes all the time. In fact, it is quite probable for human beings to arrive at partially wrong or even completely wrong conclusions. For example, people used to believe that heavier objects fall faster than lighter objects, which is a wrong conclusion. Another example is that people thought Newton's laws of motion are universally true in any case, but it turned out to be suitable only to macroscopic objects with low velocities.

On the other hand, during the long period of struggling with nature, people have summed up much experience. Such experience may not have been proved by accurate and persuadable experiments, but it does not mean that they are wrong or useless. For example, people have known for long that light travels along strait lines, and all right angles are equal to each other.

One can see that, as has been stated above, it is not guaranteed to be absolutely true even if a conclusion is drawn through scientific methods. Besides, it is not guaranteed to be absolutely wrong even if a conclusion is drawn through methods that are not scientific or not scientific enough. Let us view a conclusion on two aspects here: how and what. In other words, by what means we arrive at the conclusion, and what the conclusion itself is. Since it is not reliable for us to judge the value of a conclusion according to the way we arrive at it, it will be natural for us to change to the other aspect, that is to say, according to the conclusion itself.

Here, what we care about is whether a conclusion is relatively true ${ }^{6}$ in a knowledge system, namely, we do not really care about whether it is absolutely true ${ }^{7}$ in all knowledge systems. If a conclusion is not relatively true in a knowledge system, i.e., if it is not repeatable in this knowledge system, then people in different places and at

\footnotetext{
${ }^{5}$ Namely, we only care about the repeatability of conclusions but not their correctness here.

6 A conclusion in a knowledge system is relatively true if it is repeatable in this knowledge system.

7 If a conclusion is relatively true in a knowledge system, and this knowledge system is relatable to another knowledge system, then the conclusion is also relatively true in the second knowledge system, according to the definition of relatable. Further more, a conclusion is absolutely true if it is relatively true in all knowledge systems.
} 
different times are not guaranteed to arrive at the same conclusion, even though they start from the same axioms or hypotheses, and use the same reasoning rules. We view such a conclusion as useless, as reasoning basing on this conclusion means arriving at more unrepeatable conclusions, which damages the soundness and stableness of a knowledge system.

If a conclusion is repeatable, or equally speaking, relatively true, in a knowledge system, then it means that the conclusion has universal meanings at least in this knowledge system, which makes it valuable and useful at least therein. Further more, whether the conclusion is relatively true in another knowledge system depends on whether the original knowledge system is relatable to the second one, and whether the conclusion is absolutely true depends on whether the knowledge system is relatable to all other knowledge systems.

As a result, we take whether a conclusion is repeatable in a knowledge system as the criterion for judging whether it is valuable in this knowledge system. We call such a criterion the criterion of relatively true property. Further more, the problem of judging whether a conclusion is absolutely true turns out to be a problem of determining the relationship between knowledge systems, namely, whether they are relatable to each other. In this sense, knowledge systems are unified on the aspect of judging the value of conclusions.

\section{Why such a Criterion Is Reasonable}

One can see that this criterion is reasonable if we restrict our scope to one specific knowledge system without caring about the relationship between different knowledge systems. We take geometry as an example to discuss such a case in detail here.

Geometry, as an important branch of mathematics, is in fact made up of three different theories, which are Euclidean geometry, Lobachevskian geometry, and Riemannian geometry, among which Euclidean geometry is the one that is most widely known.

All these three types of geometry have five axioms, among which the first four are the same: two points determine a line, a line extends infinitely, take a point as center and a line segment as radius we can draw a circle, all right angles are equal. But they are different from each other on the fifth axiom. Euclidean geometry states that given a line and point outside the line within a two-dimensional plane, there exists exactly one line through the point that does not intersect with the given line. But Lobachevskian geometry claims that there are infinitely many such lines, while in Riemannian geometry there is no such line ${ }^{8}$.

Those three types of geometry are all consistent within themselves, although they are based on three different sets of axioms. We view these three types of geometry as three different knowledge systems, and for each of them the conclusions are relatively true within themselves. Note that the relatively truth here is not only repeatability, but also absolute repeatability. This is because people at different places or in different times are able to draw the same conclusions in each of these knowledge systems as long as they start from the same axioms and follow the same reasoning rules. In fact, all that we need to do is writing the proofs on a piece of paper, and that is enough.

As a result, this criterion is reasonable when used to judge whether a conclusion is valuable in a single knowledge system. Moreover, in such rigorous systems as mathematics, the criterion is not only reasonable but also practical, which depends on the fact that conclusions in such knowledge systems are absolutely repeatable.

\footnotetext{
${ }^{8}$ Lobachevskian geometry is also called Hyperbolic geometry, and Riemannian geometry is also called Elliptic geometry.
} 


\section{A Knowledge System Is Valuable if It Satisfies This Judging Criterion}

A knowledge system is valuable at least to its community of believers ${ }^{9}$ as long as it satisfies the criterion of relatively true property. Further more, whether this knowledge system is valuable to community of believers of another knowledge system depends on whether it is relatable to that knowledge system, namely, whether there exists medium methods to transform conclusions from this knowledge system to another one. If this is true, the first knowledge system will also be valuable to the community of believers of the second one, as long as they believe in these medium methods for transformation. Moreover, these two knowledge systems will be valuable to communities of believers of each other if they are relatable to each other. To be more intuitional, a knowledge system is valuable at least to its community of believers, and if we can find a set of methods to make two knowledge systems relatable to each other, then both of these two knowledge systems' community of believers expand, by absorbing each other's believers.

This has also been obviously verified by geometry. In fact, Absolute geometry introduced by Janos Bolyai in 1832 has been playing the role of medium method to make the three types of geometries relatable to each other. In Absolute geometry, the three types of geometry correspond to curvatures zero, negative numbers, and positive numbers. Before Absolute geometry was proposed, mathematicians hesitated to believe that such three types of geometry hold at the same time, resulting in three communities of believers, corresponding to the three different sets of axioms. But things are different when we found such a medium method as Absolute geometry, which merged those three communities of believers to one. This is essentially why mathematicians accept all these three types of geometry nowadays, and it is a good example of how community of believers of a knowledge system expands.

In fact, we can view a knowledge system as a kind of language to communicate with each other for its community of believers. In this sense, mathematics is a language for mathematicians and all those persons who believe in the axioms and rules of mathematics; natural science such as physics, chemistry, and biology are languages for human beings and nature; economics and other social sciences are languages for human beings and the society they construct.

For example, we say that the reason why Euclidean geometry is valuable for those people who believe in the basic axioms of Euclidean geometry and follow its reasoning rules is that they can communicate with each other with such a language and create new theorems without worrying about unexpected conflicts ${ }^{10}$. Besides, these theorems can also be reflected intuitionally in real world, which made a great contribution to expanding its community of believers to ordinary people without specific mathematical knowledge in human history.

We say that Riemannian geometry is also valuable to those people who believe in the Riemannian fifth axiom, although this geometry cannot be reflected intuitionally in real world for the difficulty people encounter to imagine that there is no parallel line. Such value is mainly reflected in two aspects. First, the believers can discuss and create new conclusions in this knowledge system without worrying about its repeatability, because conclusions that are relatively true in this knowledge system must be absolutely repeatable. Second, it is quite

\footnotetext{
${ }^{9}$ Community of believers of a knowledge system is such a group of people or objects that believe in the axioms or hypotheses of the knowledge system and follow the reasoning rules of this knowledge system. Note that a community of believers does not necessarily consist of persons only. For example, if physics is a real description of nature, we view nature as a member of the community of believers to the knowledge system of physics.

${ }^{10}$ Unexpected conflict means conflict that occurs even though we start from the same axioms and follow the same reasoning rules.
} 
probable that there exist other knowledge systems relatable to this knowledge system, although they may not have been discovered or proposed yet. In fact, when Riemannian geometry was first proposed, people did not believe that it could be useful in practice. However, several decades of years later, Albert Einstein's general theory of relativity made things different. General theory of relativity supposes that spacetime is not evenly distributed, which can only be explained with Riemannian geometry. In this way, the knowledge system of Riemannian geometry turned out to be relatable to the knowledge system of general theory of relativity, thus resulting in the expand of its community of believers.

Another typical example is the relationship between mathematics and natural sciences. This criterion is capable of explaining why, at least until now, all these kinds of natural sciences such as physics, chemistry, and biology do not conflict with each other, although they were developed almost independently at the beginning. We tend to describe natural sciences as knowledge systems created by God, while mathematics as knowledge system created by human beings. The reason why the parts of knowledge about physics, chemistry, and biology that have been discovered by us do not conflict with each other, and why we prefer to modify existing models to ensure its consistency when conflict occurs, is that we have constructed mathematics as a medium knowledge system, which makes those branches of natural science relatable. And the fact that community of believers of mathematics is almost all of the human beings makes the communities of believers of natural sciences also almost all human beings, or to be more precise, have the ability of expanding to all human beings. Further more, it is potential that there exists another extraterrestrial intelligence that uses another form of mathematics as medium knowledge system to make natural sciences such as physics and chemistry relatable, where the only difference is the form of relationships.

As a result, a knowledge system is valuable as long as it satisfies the criterion that its conclusions are relatively true in itself. Such value may be some intuitional practical value, or the value that it can be used as a language for its community of believers to communicate with each other, or even the value that it may be relatable to a knowledge system that has not been discovered or proposed. And all these kinds of value are based on the fact that they satisfy the criterion that their conclusions are relatively true at least.

\section{About Science and Superstition}

In this section, we take Fengshui as an example to see how the criterion of relatively true property above can be used in practice. Generally speaking, Fengshui is a knowledge system about how the ancients view nature and the environment they lived in. Although a few of the conclusions in this knowledge system have been proved to be reasonable to some extent, most of them are regarded to be subjectively assumed without any basis, which made people tend to refuse to accept the hypotheses and reasoning rules of Fengshui, and classify it to the category of superstitions.

Let us analyze the knowledge system of Fengshui first. As a knowledge system, its axiom is that the universe is made up of five basic elements, which are metal, wood, water, fire, and earth. It has a variety of reasoning rules, for example, Qi rides the wind and scatters, but is retained when encountering water ${ }^{11}$. Now, we do not care about the relationship of this knowledge system with other natural science knowledge systems such as physics and

11 There are detailed descriptions of all reasoning rules in the book Zangshu (Book of Burial) by GUO Pu of the Jin Dynasty. This would be a good reference if the reader were really interested in this ancient Chinese system. 
chemistry. We only care about whether conclusions are relatively true in the knowledge system of Fengshui itself. That is to say, what we care about is whether different people can arrive at the same conclusions if they start from the same axioms and follow the same reasoning rules. A conclusion here may be that whether a natural environment or the orientation of a building is good or not. If they are able to arrive at the same conclusions given the same conditions, we say that this knowledge system is valuable to its community of believers.

Fortunately, it is real that conclusions in this knowledge system of Fengshui are relatively true, which is proved by the utilization of Fengshui in archeology and history studies. In archeology for example, as we know that the ancients decided the orientations of palaces and tombs according to the knowledge system of Fengshui, we can also arrive at the same conclusions about how they were constructed if we deduce the orientations of ancient tombs according to the axioms and reasoning rules in the same knowledge system when digging at a site, which will be very helpful for archeologists to find the historical relics buried underground ${ }^{12}$. In this example, we do not care about whether the knowledge system of Fengshui is relatable to other knowledge systems that seem to be more scientific such as physics and chemistry, but it is still very valuable at least to archeologists. The essential reason here is that conclusions in this knowledge system are repeatable, or more precisely, relatively true, at least to a large extent.

Further more, the problem that whether the community of believers of this knowledge system of Fengshui can be expanded depends on whether we can find some medium methods to make this knowledge system relatable to other knowledge systems such as physics, at least partially. If so, the community of believers of Fengshui expands by absorbing the community of believers of physics, which is almost the whole mankind.

This has been verified by the fact that many conclusions in Fengshui have been explained successfully in scientific aspects to some extent. For example, a building is considered to be particularly good if it faces south with a mountain in the back as well as a river in the front. Experts from many different fields including psychology, geophysics, and architectonics ${ }^{13}$ have demonstrated this point of view. Psychologists point out that with a mountain in the back and a river in the front, people will be given a psychological suggestion of being both safe in the back and lively in the front. On the contrary, if a building faces a mountain in the front and leans on a river in the back, people will be given a psychological suggestion of being both congesting in the front and dangerous in the back. At the same time, geophysicists point out that it is reasonable for buildings in the Northern Hemisphere to face south as this is benefit for both gathering daylight and defending monsoon in winter. As a result, community of believers of the knowledge system Fengshui expands in this aspect. The essential reason here is that we can find some medium methods to make Fengshui at least partially relatable to knowledge systems that already have their own community of believers.

To make a summarization, we do not need a clear boundary between science and superstition with this criterion for judging the value of knowledge systems, and we even do not need to discuss what science is and what superstition is. All that we need to care about is whether a knowledge system is relatively true, namely, whether its conclusions are repeatable in this knowledge system itself. We can be sure that this knowledge system

\footnotetext{
12 Jack Finegan's book The Archeology of World Religions (1956) is a good reference for the study of the utilization of various kinds of Fengshui-like knowledge systems in archeology.

13 Much more detailed discussion is presented in Suhana Lim and Bruce Bentley’s Designing a Healthy Practice with Fengshui (Health traditions, Vol. 5-1).
} 
is valuable at least to its community of believers as long as this property holds, just as what we have seen in the example of the three types of geometry above. As for the problem of whether a knowledge system has larger value, we say that this is essentially a problem of whether this knowledge system is relatable to other knowledge systems, thus to expand its community of believers.

\section{Conclusions}

In this paper, we started from the relationships between mathematics and Fengshui, and proposed the criterion of relatively true property for judging whether a conclusion is valuable in a knowledge system. We demonstrated that such a criterion is valuable, and knowledge systems satisfying this criterion are also valuable. In the end, we took Fengshui in archeology as an example to show the role that the criterion plays in practice.

In conclusion, the core points of view of this paper are: a knowledge system that satisfies the criterion of relatively true property is valuable at least to its community of believers, and the problem of whether a knowledge system has larger value is essentially a problem of whether it is relatable to other knowledge systems, thus to expand its community of believers.

\section{References}

Eder, M. (2000). Views of Euclid's parallel postulate in ancient Greece and in Medieval Islam. History of mathematics. Newark: Rutgers University Press.

Ernest, P. (1996). Is mathematics discovered or invented? New angles on old rules. Times higher educational supplement. London:

TSL Education Ltd..

Finegan, J. (1965). The archeology of world religions (Vol. 2). Princeton: Princeton University Press.

Kuhn, T. (1962). The structure of scientific revolutions. Chicago: University of Chicago Press.

Lim, S., \& Bentley, B. (2010). Designing a healthy practice with Fengshui. The lantern (Vol. 5-1, pp. 22-24). Carlton: The Lantern. LIU, L. (2004). The Chinese Neolithic: Trajectories to early states. Cambridge: Cambridge University Press.

Pickering, A. (Ed.). (1992). Science as practice and culture. Chicago: University of Chicago Press.

Shermer, M. (2002). The Skeptic dictionary of pseudoscience (Vol. 2). Santa Barbara: ABC-CLIO, Inc..

SUN, X. (2000). Crossing the boundaries between heaven and man: Astronomy in ancient China. In H. Selin (Ed.), Astronomy across cultures: The history of non-western astronomy (pp. 423-454). Dordrecht: Kluwer Academic.

Thornton, S. (2006). Karl Popper. Stanford encyclopedia of philosophy. Stanford: Stanford University Press. 